\title{
Storage, retrieval, and inventory control of donor red cells in liquid nitrogen
}

\author{
R. MITCHELL AND W. MUIR
}

From the Glasgow and West of Scotland Regional Blood Transfusion Centre, Law Hospital, Carluke, Lanarkshire, Scotland

SYNOPSIS A system of identification and inventory control of donations and aliquots of human blood stored in liquid nitrogen is described. It is simple and easy to operate. Up to five adequate samples can be obtained from each donation at discrete intervals without the need to recover the main donation or, as is so often the case, the only single aliquot available.

Long-term storage of human red cells in liquid nitrogen is now established in this country and has been practised abroad (Turner, 1970). Introduction of the low glycerol-rapid freeze method of Krijnen, Kuivenhoven, and De Wit (1971) has presented us with problems of storage of donations and aliquots in a system which allows their accurate identification and subsequent retrieval and recovery for transReceived for publication 22 December 1971.

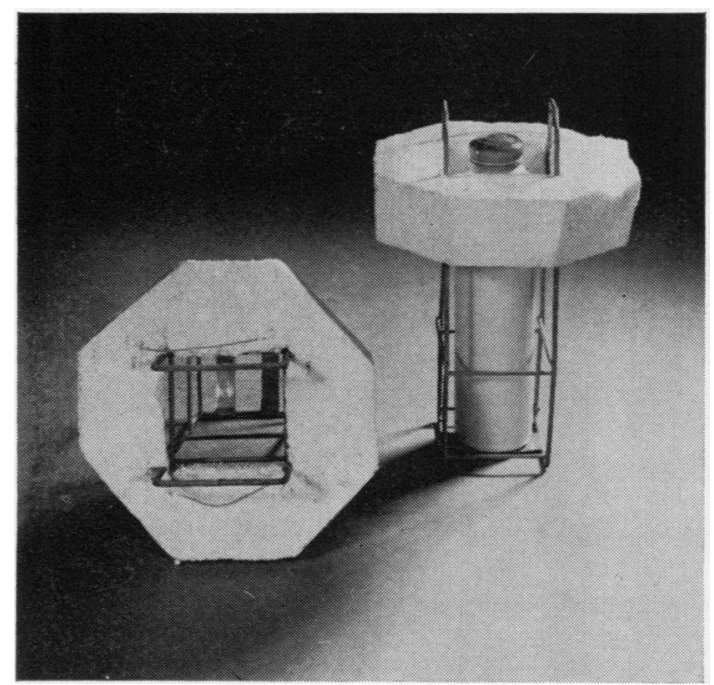

Fig. 1.

Fig. 1 System for floating cans during freezing.

Fig. 2 Carrying rack for storage in main dewar. fusion. We report here on our methods which are easy to operate and efficient in use.

Five hundred $\mathrm{ml}$ glycerolized deplasmatized blood is first frozen in a small dewar using a flotation collar of polystyrene to maintain the liquid nitrogen at the shoulders of the aluminium can (Fig. 1). Thereafter the can is stored upright in a 250 litre dewar with its neck in the gas vapour phase. The can is held in

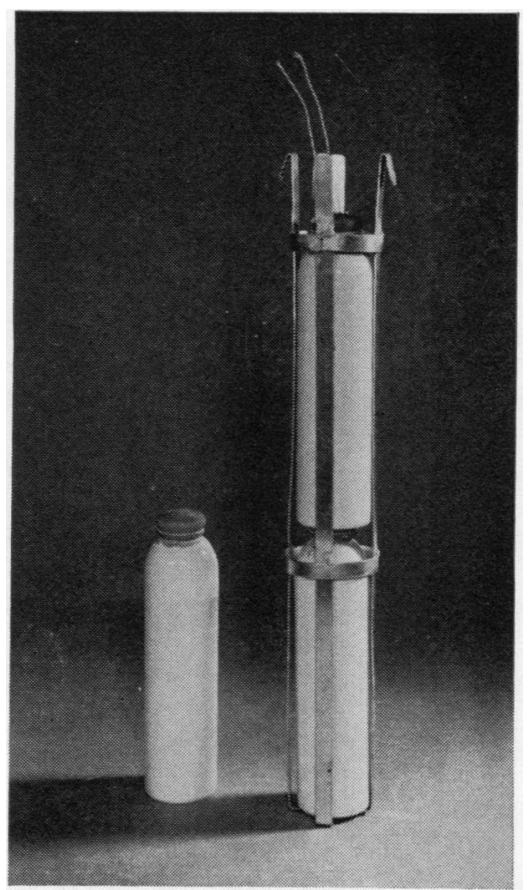

Fig. 2. 


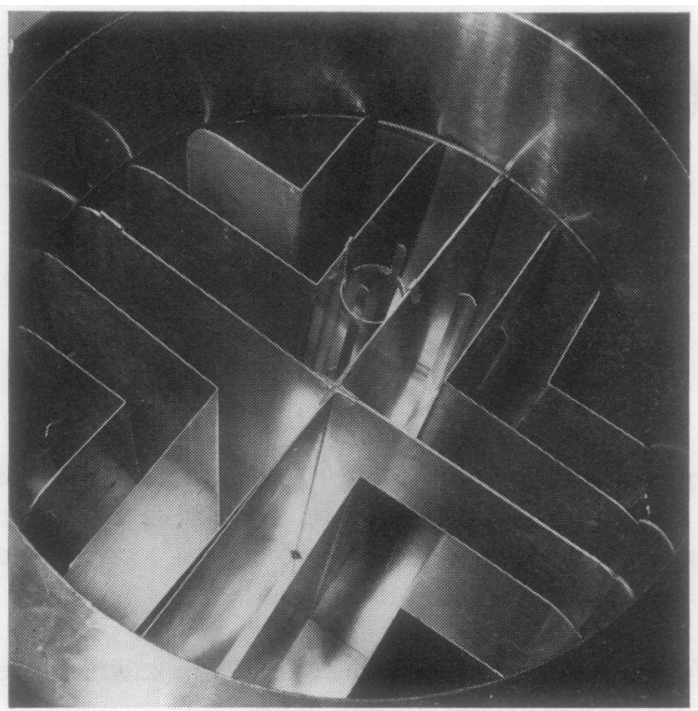

Fig. 3 Divided storage dewar.

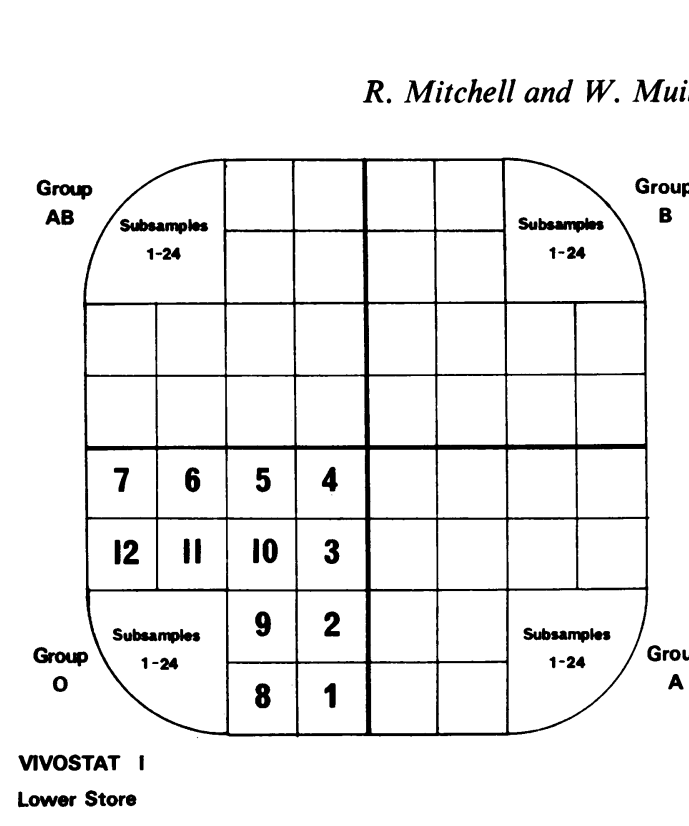

Fig. 4 Liquid nitrogen numbering system.

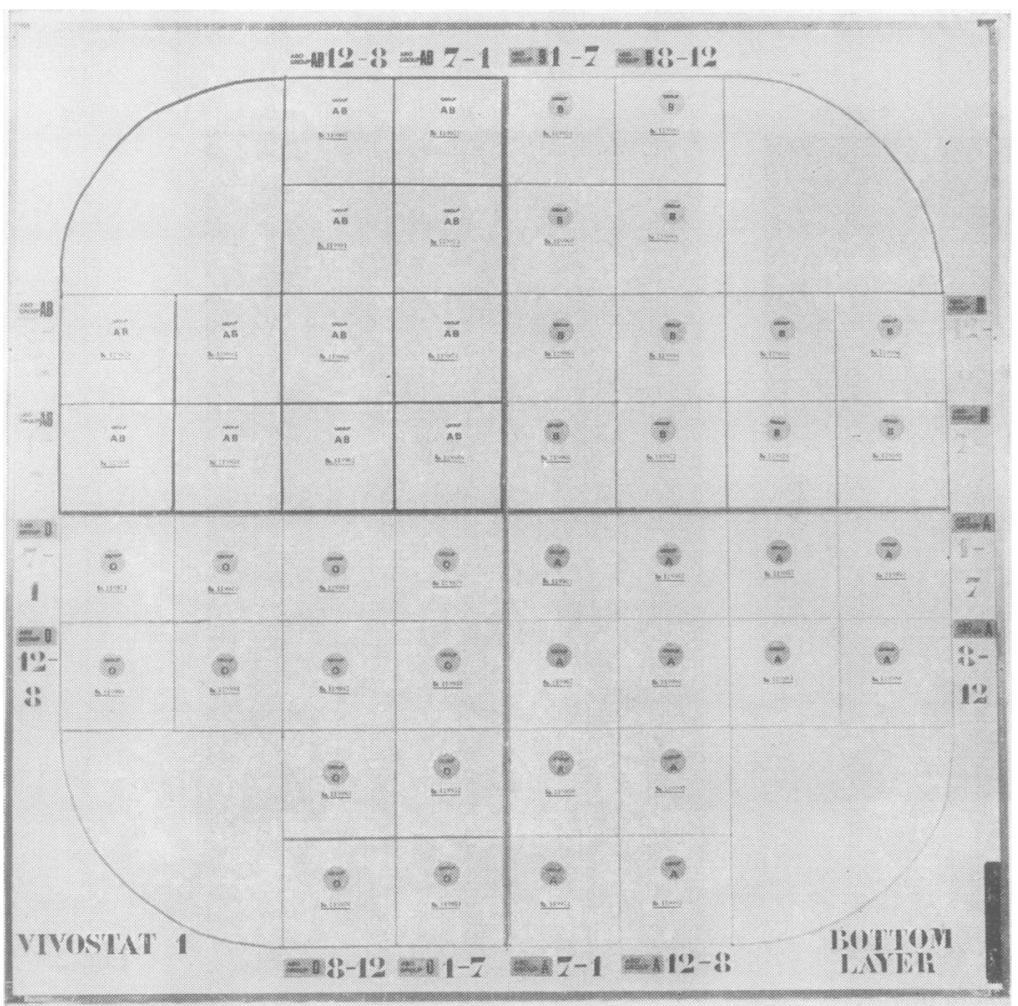

Fig. 5 Inventory occupancy board. 
position by a light aluminium carrying rack sufficiently long to allow the storage of two cans, the base of the uppermost resting on the rubber closure of the lowermost. Each can bears a copper wire secured at its neck for easy handling (Fig. 2). Each can holder has two hooks at the upper end which settle into more rigid aluminium right-angled inserts. These are free standing on the floor of the dewar and of sufficient depth to keep the lowest can shoulder above the liquid nitrogen level $(22.5 \mathrm{~cm})$. These insert panels are set $7 \mathrm{~cm}$ apart parallel to each other and effectively divide the dewar into a series of four segments each with its own compartments (Fig. 3).

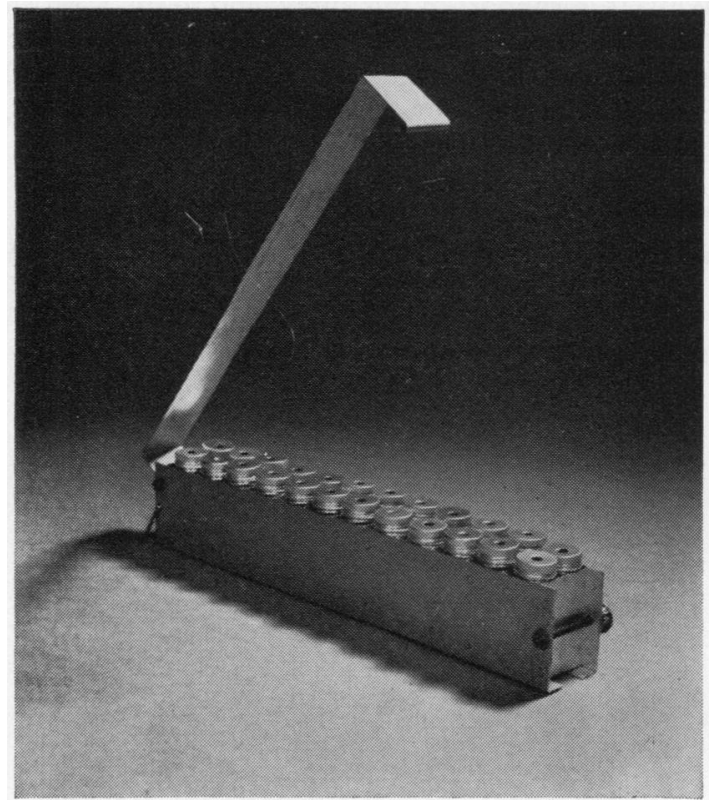

Fig. 6.
Spacer clips ensure that a rigid structure is maintained. Using this system, it is possible to label each segment with any code, eg, group $A, B, A B$, and $O$. Each position in the group segment can be allocated a number, from 1 to 12 for the bottom row and from 13 to 24 for the top row (Fig. 4). If this position number is then used as the liquid nitrogen identification number, any donation can be located immediately. Using large dewars with closed split lids only the appropriate half need be opened to allow access to the required area.

To complement the storage system within the dewar, a separate inventory chart board was drawn showing upper and lower levels of each segment. The occupancy of any position is shown by sticking the appropriate 1 in. circular coloured group disc bearing the donor's group and registered donation number over the position on the chart board (Fig. 5). Standard international colour code blood transfusion discs are used and the chart drawn on a convenient board, eg, Magiboard. A separate punch card system and work sheet for all donors is kept showing this 'liquid nitrogen number' and all other information.

At the outer circumference of each segment there is a space left in the design. This can conveniently be used for storing pilot samples for subsampling compatibility test specimens and research subsamples without the need to remove the whole donation. Small $20 \mathrm{~mm} \times 65 \mathrm{~mm}$ extruded aluminium cans with perforated screw-cap lids can be stored in a vertical rack which is divided into 12 slots, each slot taking two cans. The cans are secured in position by means of a long flip-over lid secured with a screw (Fig. 6). Each rack therefore holds 24 cans, numbered 1-24 to correspond with the donation position numbers and inventory chart numbers.

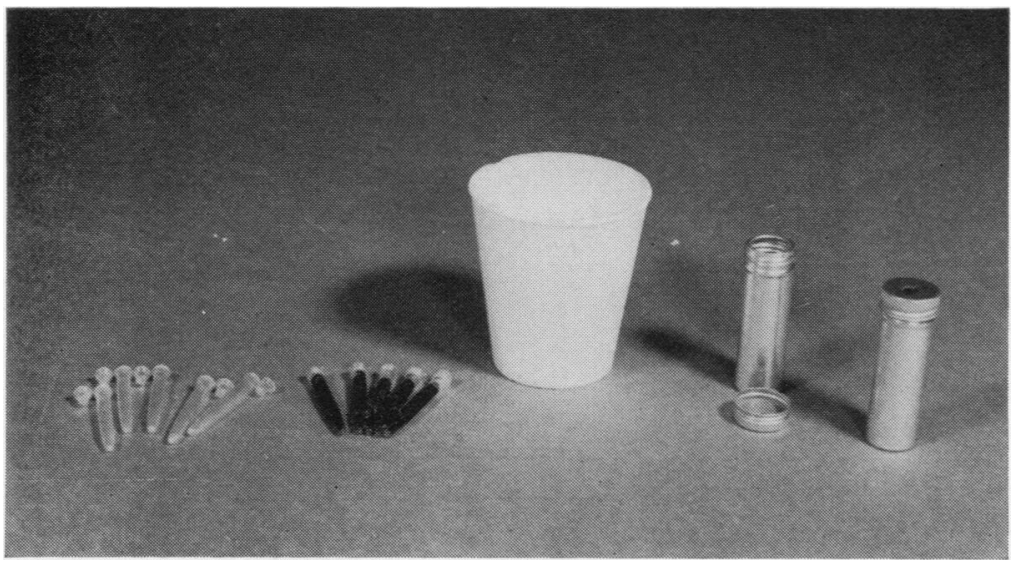

Fig. 6 Storage rack for aliquots in main dewar.

Fig. 7 Freezing of glycerolized aliquots of donor red cells.

Fig. 7. 
Each can is capable of holding five stoppered polyethylene microtubes (Beckman-Riic Ltd). Glycerolized blood collected from the residue in the donor unit before transfer to the main storage can is placed with a Pasteur pipette into each numbered microtube. Each microtube holds $0.4 \mathrm{ml}$ of glycerolized blood. The stoppers are inserted and the microtubes dropped into a small $(100 \mathrm{ml})$ volume of liquid nitrogen (Fig. 7). Cooling is rapid and 'boil off' is quiescent within one minute. The microtubes are then easily transferred to the labelled, numbered 20 $\times 65 \mathrm{~mm}$ cans for storage as described above.

To recover washed red cells suitable for matching tests and research the microtube is unstoppered and placed in $15 \mathrm{ml}$ prewarmed sorbitol/saline where with inversion, rapid thawing and release of red cells into

\begin{tabular}{lll}
\hline & ABO & $\mathbf{L e}^{\mathbf{a}}$ \\
CcDEe & $\mathbf{L e}^{\mathbf{b}}$ \\
MNSs & $\mathbf{F y}^{\mathbf{a}}$ \\
$\mathbf{C l}$ & $\mathbf{J k}^{\mathbf{a}}$ \\
$\mathbf{P}$ & $\mathbf{K p}^{\mathbf{a}}$ \\
& Kell & $\mathbf{L u}^{\mathbf{a}}$ \\
Cellano & $\mathbf{W r}^{\mathbf{a}}$ \\
\hline
\end{tabular}

Table Antigens tested before and after thawing the wash fluid is obtained within two minutes (first Krijnen wash). Two physiological saline washes. follow in succession. At least $5 \mathrm{ml}$ of a $10 \%$ cell suspension is thus easily recovered. We have tested $\stackrel{\text { ? }}{?}$ thawed aliquots in this manner over a period of six? months with no obvious loss of antigenicity (Table).

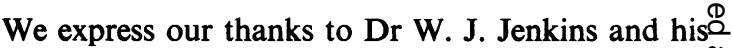
colleagues at the North-East Metropolitan Regionalis Blood Transfusion Centre, Brentwood, for adviceand encouragement on the use of the extrudedaluminium (Spembly) canisters; to the Scottishw Home and Health Department for a new medical development grant; to our Regional Director, Dr J? Wallace in support of this study, and to the Scottishis National Blood Transfusion Association for time's and facilities. Our medical, scientific and technicalo colleagues have our thanks for their interest ${ }_{0}^{2}$ enthusiasm, and talented help.

References

Krijnen, H. W., Kuivenhoven, A. C. J., and De Wit, J. J. Fr. M. (1971). Preservation of tlood cells in the frozen state. In $\overrightarrow{0}$ Modern Problems of Blcod Preservation, edited by W.Spielmann, and S. Seidl, pp. 176-183. Fischer, Stuttgart.

Turner, A. R. (1970). Frozen Blood. A Review of the Literature 1949 1968. Gordon and Breach, New York. 